\title{
Theme issue on adaptation and personalization for ubiquitous computing
}

\author{
Zhiwen Yu $\cdot$ Doreen Cheng $\cdot$ Ismail Khalil $\cdot$ \\ Judy Kay $\cdot$ Dominikus Heckmann
}

Published online: 2 August 2011

(C) Springer-Verlag London Limited 2011

Ubiquitous computing is a human-centered paradigm that aims to provide users with adaptive and personalized services according to their surrounding context. Adaptation and personalization technologies are thus an important basis of ubiquitous computing. They are also the core for realizing context awareness in pervasive service provisioning. In ubiquitous computing environments, people are surrounded by many networked computers, both fixed (e.g., PCs, TVs) and mobile devices such as PDAs, cellular phones, etc. People are increasingly able to access their desired content, anytime, anywhere using the available devices. To offer the right information to users at the right time, right place and in the right way is challenging for many reasons, such as varying user interests,

\section{Z. Yu ( $\square)$}

School of Computer Science, Northwestern Polytechnical

University, Xian, China

e-mail: zhiwenyu@nwpu.edu.cn

D. Cheng

Computer Science Lab, Samsung R\&D Center,

San Jose, CA 95134, USA

e-mail: doreen.c@samsung.com

\section{Khalil}

Institute of Telecooperation, Johannes Kepler University Linz,

Linz, Austria

e-mail: ismail.khalil@jku.at

\section{J. Kay}

School of Information Technologies, University of Sydney,

Sydney, NSW 2006, Australia

e-mail: judy.kay@sydney.edu.au

D. Heckmann

Intelligent User Interfaces Group, DFKI, Saarbrücken, Germany

e-mail: heckmann@dfki.de heterogeneous environments and devices, dynamic networks, information overload, user privacy, and so on.

This theme issue aims to explore adaptation and personalization services and technologies for ubiquitous computing. Submissions to this special issue came from an open call for papers as well as from selected papers presented at the 7th International Conference on Ubiquitous Intelligence and Computing (UIC 2010) held at Xi'an, China, October 26-29, 2010. We received a total of 26 submissions of which 8 papers were accepted after three rounds of rigorous reviews. We are grateful to the large number of reviewers who assisted us in the review process; in order to ensure high reviewing standards, three to four reviewers evaluated each paper.

The opening paper of this special issue, "Social itinerary recommendation from user-generated digital trails", authored by Hyoseok Yoon, Yu Zheng, Xing Xie, and Woontack Woo received the best paper award of UIC 2010. The paper addresses the problem of planning travel to unfamiliar regions for novice travelers. It proposes recommending a social itinerary by learning from multiple user-generated digital trails, such as GPS trajectories of residents and travel experts. It describes an itinerary model in terms of attributes extracted from user-generated GPS trajectories, and a social itinerary recommendation framework that can find and rank itinerary candidates. It also reports the evaluation results using a large set of usergenerated GPS trajectories collected from Beijing, China.

The second paper, "TruBeRepec: a trust-behavior-based reputation and recommender system for mobile applications", by Zheng Yan, Peng Zhang, and Robert H. Deng, examines the trustworthiness of mobile applications that are to be recommended to a user. The authors introduce a model of trust behavior for mobile applications based on the results of a large-scale user survey. Several algorithms 
were developed to evaluate individual user's trust in a mobile application through trust behavior observation, to generate the application's reputation by aggregating individual trust, and provide application recommendations based on the correlation of trust behaviors. Through simulations, the system was tested and analyzed for effectiveness, robustness, and usability, as well as privacy.

In "Context relevance assessment and exploitation in mobile recommender systems", Linas Baltrunas, Bernd Ludwig, Stefan Peer, and Francesco Ricci explore the problem of which contextual factors are important and to which degree they influence user ratings in a context-aware recommender system that uses not only user preferences, but also the contextual situation when generating relevant recommendations. The paper presents an approach for assessing and modeling the relationship between contextual factors and item ratings. For data capture, the authors simulated contextual situations and designed a methodology whereby users are asked to judge whether a contextual factor (e.g., season) influences the rating given in a certain contextual condition. Based on the analysis, a contextaware mobile recommender system that utilizes contextual factors was built.

The fourth paper, "AML: a modeling language for designing adaptive web applications", written by Roberto De Virgilio presents a conceptual model to specify complex adaptive web applications. It models the adaptation process by using a special workflow. It also models the executable elementary manipulations as a set of primitives. The model and its design process are implemented in a CASE tool, called FAWIS. The system can access a Web data source and automatically filter and adapt information delivery on the basis of the context of the client.

In the 5th paper, "Personalization for unobtrusive service interaction", Miriam Gil, Pau Giner, and Vicente Pelechano indicate that services need to be personalized according to the user needs and environmental context, avoiding service behavior from becoming overwhelming. To address this information overload problem, a method for the development of mobile services that can be personalized in terms of obtrusiveness according to the user needs and preferences, is presented. With this method, services can be developed to provide their functionality at different obtrusiveness levels depending on the user.

The sixth paper, "Personalization and user verification in wearable systems using biometric walking patterns", written by Pierluigi Casale, Oriol Pujol, and Petia Radeva presents a method for user authentication and verification using gait as a biometric and unobtrusive measure. The method has two phases. The first phase trains a general activity recognition classifier for a specific user using a small sample of the user's walking patterns. The second phase verifies whether the user is an authorized one or not, which is essentially a one-class classification problem. The authors proposed two scenarios with two different wearable systems, a custom high-performance wearable system and an Android-based commercial device, to validate the approach.

In "DIY-CDR: an ontology-based, do-it-yourself components discoverer and recommender", Yan Tang and Robert Meersman present an interesting study of using DoIt-Yourself (DIY) in the area of computer science. They present a computing environment to support users to DIY their personalized Internet-of-Things (IoT) applications. One key technique can automatically discover and recommend existing components to users. The paper adopts the Controlled Fully Automated Ontology Assisted Matching Strategy (C-FOAM) in the component discovery and recommendation module. It also uses semantic decision tables to gather user-specific decision rules and set up parameters for C-FOAM.

The last paper, "Generating recommendations for consensus negotiation in group personalization services", by Maria Salamó, Kevin McCarthy, and Barry Smyth investigates group personalization services in ubiquitous computing environments with an example of ubiquitous commerce. One of the challenges a group recommender system must cope with is the potentially conflicting preferences of multiple users when selecting items for recommendation. This paper focuses on how individual user models can be aggregated to reach a consensus on recommendations. It describes and evaluated nine different consensus strategies. The results showed that the performance is significantly different among strategies.

The eight selected papers capture some of the state-ofthe-art research issues in personalization, adaptation and recommendation, in ubiquitous computing. We hope that the key issues presented here, such as itinerary, application and component recommendation, trustworthiness and unobtrusiveness in recommendation, context-aware recommenders, content adaptation, and group personalization, will advance the discussion in the community toward future ubiquitous personalized systems. We would like to thank Prof. Peter Thomas, the Editor-in-Chief, for his great support and work throughout the whole publication process. We are also grateful to the referees for their professional and timely reviews. Last, but not least, we would like to express our appreciation to all the authors who submitted papers to this theme issue. 